\title{
MRI Analysis of the Cerebellum in Bipolar Disorder: A Pilot Study
}

\author{
Melissa P. DelBello, M.D., Stephen M. Strakowski, M.D., Molly E. Zimmerman, B.A., \\ John M. Hawkins, M.D., and Kenji W. Sax, Ph.D.
}

Since qualitative CT studies have suggested decreased cerebellar size in patients with bipolar disorder, we performed a quantitative analysis of the cerebellum in patients with bipolar disorder to determine whether highresolution, thin slice magnetic resonance imaging (MRI) morphometry would reveal similar results. Bipolar patients hospitalized for a first manic episode $(\mathrm{n}=16)$, bipolar patients with prior manic episodes hospitalized for a manic episode $(\mathrm{n}=14)$, and normal volunteers $(\mathrm{n}=15)$ matched for age, sex, race, and education were recruited and anatomic brain scans were acquired using a Picker 1.5 Tesla MRI scanner. Right and left cerebellar hemisphere volumes and vermal areas V1 (lobules I-V), V2 (lobules VI-VII), and V3 (lobules VIII-X) were measured. ANCOVA comparing each ROI, adjusting for race, sex, age, total cerebral volume, and substance abuse duration, revealed a significant group effect for vermal $V 3$ area. Specifically, V3 area was significantly smaller in multiple-episode patients than in first-episode patients or healthy volunteers. Number of previous episodes of depression may contribute to this finding. These results suggest that cerebellar vermal atrophy may be a later neurodegenerative event in patients with bipolar disorder who have had multiple affective episodes. The confounding effects of medications are considered. [Neuropsychopharmacology 21:63-68, 1999] (C) 1999 American College of Neuropsychopharmacology. Published by Elsevier Science Inc.
KEY WORDS: MRI; Bipolar disorder; Cerebellum; Vermis

Although traditionally regarded as a regulator of motor function, the cerebellum has also been found to project to regions of the brain that modulate mood (Anand et al. 1959; Heath et al. 1978; Courchesne et al. 1989). Several case reports have identified cerebellar atrophy in patients with mood dysregulation and in patients with bipolar disorder (Cutting 1976; Hamilton et al. 1983; Joseph et al. 1985; Yadalam et al. 1985; Jurjus et al. 1994).

From the Bipolar and Psychotic Disorders Research Program, Department of Psychiatry, University of Cincinnati College of Medicine, Cincinnati, Ohio.

Address correspondence to: Dr. DelBello, Department of Psychiatry, University of Cincinnati College of Medicine, 231 Bethesda Ave., P.O. Box 670559, Cincinnati, OH 45267-0559.

Received September 28, 1998; revised January 25, 1999; accepted February 19, 1999.
Moreover, several studies have found abnormal cerebellar anatomy in patients with affective disorders (reviewed in Soares and Mann 1997). Two studies reported a greater rate of cerebellar atrophy in manic patients than in patients with schizophrenia or normal controls (Nasrallah et al. 1981, 1982). Additionally, Yates et al. (1987) found a greater rate of cerebellar atrophy in patients with bipolar disorder who were over fifty years old, but not in younger bipolar patients compared with healthy volunteers. Lippmann et al. (1982) found bipolar patients with co-occurring alcohol abuse had increased rates of both vermal atrophy and cerebellar hemisphere atrophy compared with normal healthy volunteers, suggesting that alcohol abuse may contribute to abnormal cerebellar anatomy in bipolar patients. Rieder et al. (1983) found no difference in cerebellar size among patients with bipolar disorder, schizophrenia, and schizoaffective disorder. Results of these contradic- 
tory studies are limited, since they qualitatively assessed cerebellar size based on gross abnormalities found on CT scans. Quantitative measurements and adequate differentiation between cerebellar hemispheres and vermis were not performed.

Despite numerous studies suggesting an association between cerebellar atrophy and bipolar disorder, there are no quantitative analyses of the cerebellum in patients with mania using MRI morphometry (Soares and Mann 1997). The aim of this study was to morphometrically assess the cerebellum of patients with bipolar disorder using high-resolution, thin slice, MRI, which allows for superior visualization of cerebellar anatomy.

Previous investigators have found that lithium toxicity (Donaldson and Cuningham 1983; Kores and Lader 1997), depression (Shah et al. 1992; Escalona et al. 1993), and alcohol use (Lippmann et al. 1982) are associated with smaller cerebellar size. Therefore, cerebellar size may be associated with chronic affective illness or confounding variables associated with chronicity, such as number of previous episodes of depression, substance use, and medication effects. Yates et al. (1987) found that multiple-episode bipolar patients had a greater rate of cerebellar atrophy that healthy volunteers. Studying bipolar patients at the onset of their illnesses may eliminate confounding variables associated with chronic affective illness and allow identification of pre-existing neuroanatomic abnormalities in patients at their firstmanic episode. Thus, in order to evaluate the longitudinal effects of bipolar disorder on the cerebellum, we divided bipolar patients into two groups similar in age; those hospitalized for their first manic episode and those hospitalized for mania who were previously diagnosed with bipolar disorder. Based on cerebellar neuroanatomic pathways and previous studies, we hypothesized that patients with multiple-episode bipolar disorder would have smaller cerebellar sizes.

\section{METHODS}

\section{Subjects}

Bipolar patients hospitalized for a first manic episode $(n=16)$ and bipolar patients with prior manic episodes also hospitalized for mania $(n=14)$ were recruited from consecutive admissions to the inpatient Psychobiology Unit at The University of Cincinnati (Sax et al. 1999; Strakowski et al. 1999). All patients were between the ages of 18 and 45 years and met DSM-III-R criteria for bipolar disorder, currently manic, or mixed. Patients were excluded if their manic symptoms were secondary to substance intoxication or withdrawal, if they had any lifetime substance dependence, or a lifetime substance abuse history of greater than ten years duration. Diagnoses were made using the Structured Clinical Interview for DSM-III-R-Patient edition (SCID-P; Spitzer et al. 1990) performed by psychiatrists with established interrater diagnostic reliability (kappa $=.94$; Strakowski et al. 1998). Retrospective chart reviews were used to assess lifetime duration of lithium exposure, as there are reports of lithium contributing to cerebellar degeneration (Donaldson and Cuningham 1983; Kores and Lader 1997).

Healthy volunteers $(n=15)$ between the ages of 18 and 45 years, group- matched for age, education, race, and sex were recruited from the community and screened using the SCID-P. Controls were excluded for a history of any psychiatric disorder, any lifetime substance dependence, lifetime substance abuse of greater than ten years, or any first-degree relative with a psychiatric disorder.

Additional exclusion criteria for all groups were the presence of any major medical or neurological disorder, a history of head trauma, any contraindication to receiving an MRI, a diagnosis of mental retardation, or a positive pregnancy test. After complete description of the study to the subjects, written informed consent was obtained.

\section{MRI Procedures}

All subjects were scanned on the same Picker 1.5 Tesla scanner (Picker International, Cleveland, Ohio) using a RF-spoiled FAST 3-D acquisition technique (TR $=22$ msec; $\mathrm{TE}=7 \mathrm{msec}$; flip angle $=25$ degrees; field of view $=24 \mathrm{~cm}$; and matrix $=256 \times 256$ pixels). The 3-D images were then reformatted and resliced into $1 \mathrm{~mm}$ thick coronal slices. Images were digitally transferred to a Macintosh workstation, where morphometric analyses were performed using Brain Image version 2.3.3, which has the capacity to display images simultaneously in three planes (coronal, sagittal, and axial) and allows for semiautomatic region of interest (ROI) measurements. Measurements were performed by trained raters (MEZ, MPD), blind to subject identity and group. In order to measure each cerebellar region of interest, the midsagittal slice from each subject's scan was first located using all three planes. The midsagittal slice was defined as the slice where the cerebral aqueduct was the most patent, only the vermis of the cerebellum (no hemispheres) was viewed, and the posterior commisure was most clearly visualized. The images were then realigned parallel to the floor of the fourth ventricle. Left and right cerebellar hemisphere volumes were calculated separately by summing the hemisphere areas in the axial plane in approximately 30-40 consecutive slices and then multiplying by the slice thickness (1 $\mathrm{mm})$. The cerebellar hemisphere areas were manually traced in order to isolate them from surrounding structures. The vermis, cerebellar peduncles, and fourth ventricle were excluded from measurements, whereas the hemispheric gray matter, cerebellar tonsils, and corpus 
medullare were included. The ROI was then measured using a thresholding technique (Strakowski et al. 1993, 1999).

The vermis was divided into three ROIs (Courchesne et al. 1988): V1 = lobules I-V, V2 = lobules VI-VII, and V3 = lobules VIII-X. The cerebellar tonsils were not included in measurements as they are not part of the vermis. The fourth ventricle and the primary fissure demarcated V1 (the anterior lobe). The primary fissure and the prepyramidal fissure demarcated V2 (the superior posterior lobe). The prepyramidal fissure and the fourth ventricle demarcated V3 (the inferior posterior and flocculonodular lobes). The areas of V1, V2, and V3 were measured on the realigned midsagittal slice by tracing the ROI and then using a thresholding technique. Total cerebral volumes were measured to control for differences in brain size when making comparisons (Strakowski et al. 1993, 1998). Interrater reliability was established for each structure by having two raters independently measure each ROI from 10 subjects. The interclass coefficients (ICC) for interrater reliability for the left cerebellar hemisphere was 0.94, the right hemisphere was 0.92 , V1 was 0.90 , V2 was 0.96 , V3 was 0.95 , and total cerebral volume was 0.93 .

\section{Statistical Analysis}

Statistical analyses were performed using The Statistical Analysis System (SAS) for the PC (SAS Institute, Cary, NC, 1996). For the purpose of analysis, alcohol abuse and other drug abuse duration were combined into substance abuse duration as there were no control subjects with drug abuse, and additionally, both cannabis and alcohol have been associated with smaller cerebellar size (Aasly et al. 1993). Comparisons were made between multiple-episode patients, first-episode patients and healthy volunteers for each ROI using an analysis of covariance (ANCOVA), adjusting for total cerebral volume, race, sex, age, and substance abuse duration. To assess group differences among each of the five ROIs, we applied a Bonferroni-type correction in which the level of significance was adjusted $(p=.01)$ for five different comparisons (ROIs). Each dependent variable was normally distributed (as determined by proc univariate in SAS), therefore, unranked ANCOVAs were used. Where a significant model was found least squared means tests were then performed to determine which groups significantly differed. In addition, effect size was calculated for each ROI. Effect size was based on the $F$-value obtained from the ANCOVA using the formula $f=V(k-1) \mathrm{F} / \mathrm{N}$ (Cohen 1977). A multiple regression analysis was preformed to assess the variables that based on previous studies would be hypothesized to contribute to small vermal size specifically, number of previous episodes of depression (Shah et al. 1992; Escalona et al. 1993), lithium exposure (Donaldson and
Cuningham 1983; Kores and Lader 1997) and history of substance abuse (Aasly et al. 1993). Other analyses were performed as necessary.

\section{RESULTS}

Results are presented in Table 1. Significant differences were found among the groups in substance abuse history $\left(\chi^{2}=5.8, d f=2, p=.05\right)$ and duration of substance abuse $[F(2,42)=5.0, p=.01]$. There were more multiple-episode patients who abused substances and they had a longer duration of use compared to first-episode patients and healthy volunteers. Specifically, there were $4(25 \%)$ first-episode, $8(57 \%)$ multiple-episode patients, and $2(13 \%)$ healthy volunteers who abused alcohol and $7(43 \%)$ first-episode, 6 (43\%) multiple-episode patients, and no healthy volunteers who abused drugs. All of the patients who abused drugs had abused marijuana, although one, in the first-episode group, also abused stimulants and one, in the multiple-episode group, also abused sedatives and cocaine. Pearson Correlation coefficients, however, showed that substance abuse duration was not associated with any cerebellar ROI measured in this study (highest $r=-.22, p=.15$ for area V3). Seven of the 14 multiple-episode patients had previous lithium exposure (mean $=6.0$ months, $\mathrm{SD}=14$ ). At the University of Cincinnati, most patients with bipolar disorder are treated with sodium divalproex. Within the multiple-episode group, lifetime duration of lithium exposure was not associated with any cerebellar ROI measured (highest $r=-.4, p=.1$, for area V1).

ANCOVA comparing each ROI, adjusting for race, sex, age, total cerebral volume, and substance abuse duration, revealed no differences between first-episode patients, multiple-episode patients, and healthy volunteers for right cerebellar hemisphere volume, left cerebellar hemisphere volume, V1 area, or V2 area. However, a significant group effect was observed for V3 area $[F(2,36)=7.52, p=.002]$ (Figure 1). Specifically, multiple-episode patients had significantly smaller V3 areas than both first-episode patients (least squared means, $p<.05$ ) and healthy volunteers (least squared means, $p<.05$ ). The difference in V3 area between healthy volunteers and multiple-episode, as well as, between first-episode and multiple-episode patients exhibited a large effect size of $(f)=.5$. Differences in both left and right cerebellar hemispheres exhibited an effect size of $(f)=.3$. V1 had an effect size of $(f)=.2$ and V2 had an effect size of $(f)=.1$. There were four multipleepisode patients and one first-episode patient who had abused substances within the three months prior to admission. The data were reanalyzed without these five subjects and V3 was still found to be significantly smaller in multiple-episode patients than in first-episode patients or healthy volunteers. The number of pre- 
Table 1. Demographics and MRI Morphometric Measurements in Patients with Multiple-Episode Mania, FirstEpisode Mania, and Healthy Volunteers

\begin{tabular}{|c|c|c|c|}
\hline Variable & $\begin{array}{c}\text { Multiple-Episode } \\
\text { Patients } \\
(n=14)\end{array}$ & $\begin{array}{l}\text { First-Episode Patients } \\
\qquad(n=16)\end{array}$ & $\begin{array}{l}\text { Controls } \\
(n=15)\end{array}$ \\
\hline Age, mean $(\mathrm{SD})$ years & $29(5)$ & $24(4)$ & $27(5)$ \\
\hline Sex, N (\%) women & $5(36)$ & $7(44)$ & $7(47)$ \\
\hline Race, N (\%) African American & $3(21)$ & $7(44)$ & $6(40)$ \\
\hline Education, mean $(\mathrm{SD})$ years & $13(2)$ & $13(2)$ & $14(2)$ \\
\hline History of substance abuse, $\mathrm{N}(\%)^{a}$ & $9(64)$ & $7(44)$ & $3(20)$ \\
\hline Duration of substance abuse, mean (SD) years ${ }^{b}$ & $3.6(3.2)$ & $2.0(2.9)$ & $0.5(1.2)$ \\
\hline Duration of illness, mean (SD) years ${ }^{c}$ & $8.7(5.2)$ & $2.8(3.7)$ & $\mathrm{N} / \mathrm{A}$ \\
\hline No. of previous manic episodes, mean (SD) & $4.6(4.3)$ & $0(0)$ & $\mathrm{N} / \mathrm{A}$ \\
\hline No. of previous depressive episodes, mean (SD) & $4.4(5.1)$ & $1.1(1.6)^{d}$ & $\mathrm{~N} / \mathrm{A}$ \\
\hline Months of lithium exposure, mean (SD) & $6.0(13.7)$ & $0(0)$ & $0(0)$ \\
\hline \multicolumn{4}{|l|}{ ROI, mean $\mathrm{cm}^{3}(\mathrm{SD})$} \\
\hline Total cerebrum & $1,175.9(138.2)$ & $1,126.7(104.5)$ & $1,138.8(98.4)$ \\
\hline Right cerebellum & $62.2(7.7)$ & $62.9(6.6)$ & $62.0(7.5)$ \\
\hline Left cerebellum & $62.4(6.7)$ & $63.9(7.6)$ & $63.7(7.6)$ \\
\hline \multicolumn{4}{|l|}{ ROI, mean $\mathrm{mm}^{2}(\mathrm{SD})$} \\
\hline Vermal Area 1 & $396.0(57.4)$ & $393.0(45.0)$ & $395.5(46.1)$ \\
\hline Vermal Area 2 & $261.7(46.6)$ & $276.8(38.5)$ & $257.4(44.8)$ \\
\hline Vermal $3^{e}$ & $297.1(53.3)$ & $321.2(37.9)$ & $322.9(42.6)$ \\
\hline
\end{tabular}

${ }^{a}$ Significant difference $: \chi^{2}=5.8, \mathrm{df}=2, p=.05$

${ }^{b}$ Significant difference: ANOVA: $\mathrm{F}(2,42)=5.0, p=.01$ multiple-episodes $>$ controls

${ }^{c}$ Significant difference: ANOVA; $\mathrm{F}(1,28)=14.0, p=.001$ multiple-episodes $>$ first-episodes

${ }^{d}$ One patients described depression since childhood and was not included.

${ }^{e}$ Significant difference: ANCOVA: $\mathrm{F}(2,36)=7.52, p=.002$ multiple-episodes $<$ first-episodes and controls

vious manic episodes was not significantly associated with V3 area, $(r=.06, p=.8)$. However, there was a suggestion that the number of previous episodes of depression may be associated with this region $(r=-0.5$, $p=.1)$. Since thirteen of the 16 first-episode patients had either no or only one prior depressive episode, we did not assess the effects of prior depressive episodes on cerebellar size in the first-episode patients.

Finally to extend this analysis, a multiple regression was performed to examine the contribution of variables previously shown to have an effect on vermal size to the variance of V3 area between multiple-episode bipolar patients, first-episode patients, and healthy volunteers. The multiple regression in multiple-episode patients revealed that the number of previous depressive episodes, but not substance abuse duration or duration of lithium exposure, may have contributed to the V3 area size $\left(F=3.94, \mathrm{R}^{2}=.33, p=.07\right)$.

\section{DISCUSSION}

To our knowledge, this is the first MRI morphometric study examining cerebellar differences among first and multiple episode bipolar patients and healthy volunteers. The results of our study are not consistent with CT studies that have found decreased total cerebellar size in bipolar patients (Soares and Mann 1997). Since we found no difference in V3 area between first-episode patients and healthy volunteers, the decrease in cerebellar vermal size in multiple-episode patients may be a later neurodegenerative event in patients with bipolar disorder who have had multiple affective episodes. Alternatively, these findings may reflect the effects of medication exposure on the cerebellum associated with repeated affective episodes. Previous studies have described cerebellar neurologic sequelae after lithium overdose in humans (Donaldson and Cuningham 1983; Kores and Lader 1997) and cerebellar degeneration after lithium exposure in molecular studies (Lucas et al. 1998). Although in this study lithium exposure was not associated with any of the cerebellar ROIs, future studies should continue to evaluate medication effects, specifically mood stabilizers. None of the healthy volunteer subjects had abused drugs other than alcohol, therefore, for the purpose of our analysis all substances were combined into "substance abuse duration." In future studies, it may be useful to examine effects of individual drugs on cerebellar size by recruiting healthy volunteers that abuse drugs other than alcohol. In contrast to previous studies (Shear et al. 1996, Aasly et al. 1993), none of the cerebellar ROIs were significantly correlated with substance abuse duration. One explanation for this may be that the duration of alcohol abuse 


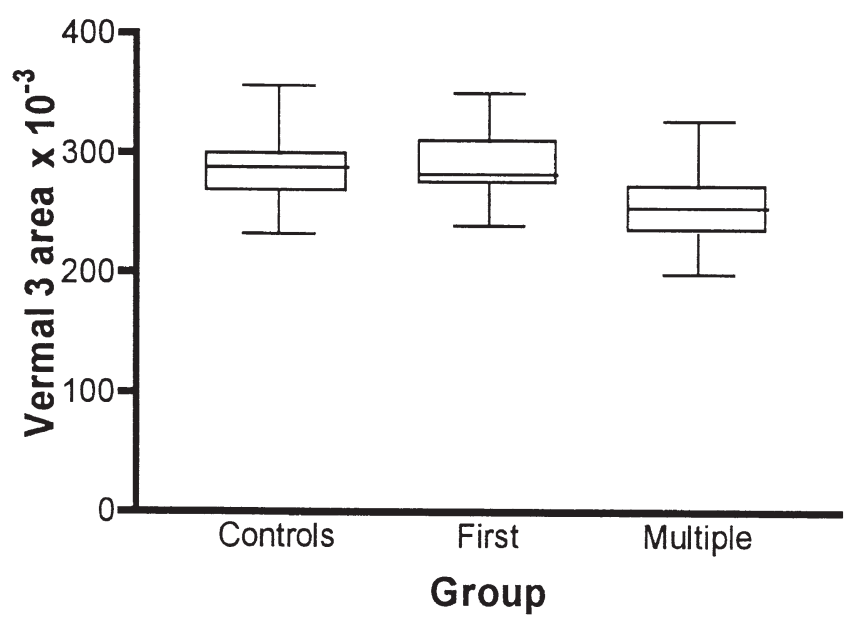

Figure 1. Adjusted cerebellar vermal 3 area vs. group

was shorter in our bipolar patient sample than in the primary substance abuser populations examined in previous studies. The number of previous episodes of depression may be largely associated with the smaller V3 area in multiple-episode patients, but this requires future study. Others (Shah et al. 1992) found that depressed patients have smaller anterior, posterior and total vermal areas compared to healthy volunteers.

Vermal area 3 consists of the inferior posterior lobe and nodulus (flocculonodular lobe) of the vermis. Several animal studies support the possibility that these regions play a role in the modulation of behavior. Anand et al. (1959) stimulated the flocculonodular lobe and the anterior and posterior portions of the vermis of dogs and found heavy projections from the flocculonodular and adjoining posterior regions of the vermis to the orbitomesial cortex, anterior cigulate gyrus, amygdala, hippocampal and dentate gyri, pyriform and periamygdaloid cortical regions, and hypothalamus, all of which are believed to regulate mood. In contrast, there was no evidence for connections of vermal region V2 to limbic structures other than the hypothalamus. Furthermore, neocerebellar hemisphere lesions in highly aggressive rhesus monkeys resulted in no change in aggressive behavior whereas animals that received lesions in the vermis and flocculonodular lobe showed no signs of aggression even after their ataxia subsided (Berman et al. 1978). Although the function of the inferior posterior cerebellar vermis and flocculonodular lobe remains largely unknown, based on these previous studies, a role in regulation of behaviors that are altered in affective illnesses, including depression and bipolar disorder may be speculated.

The findings of this study are limited by several factors. First, to more accurately reach conclusions about neuroanatomical changes that take place as bipolar disorder progresses, a prospective longitudinal study is needed. Second, confounding variables, such as medication history and IQ, may have contributed to the results. In subsequent studies, course of illness and treatment information need to be included to better understand their effects. Individual medication effects on cerebellar size should also be examined. Although in previous studies lithium toxicity has been associated with cerebellar atrophy, in our study, lithium exposure is not associated with cerebellar size. Others have found that neuroleptic exposure is not correlated with cerebellar vermal size in children with schizophrenia (Jacobsen et al. 1997). Additionally, identifying associations between cerebellar clinical findings (i.e., ataxia) and neuroanatomy may be useful.

Third, effect sizes from other ROIs showed that we may have insufficient power to detect differences. We estimate that to obtain a power $=0.8$ we would need 36 subjects in each group for the ROIs with an effect size $(f)=.3$. Finally, because of the difficulty in separating the lateral vermal boundaries from the overlying cerebellar hemispheres, a midsagittal area measurement was obtained. A volumetric measurement may provide a more valid measure of the vermis. Despite the limitations, our report suggests that the cerebellum warrants further structural and functional neuroanatomic investigation in patients with affective disorders.

\section{ACKNOWLEDGMENTS}

This study ws supported in part by NIMH grant MH54317.

\section{REFERENCES}

Aasly J, Storsaeter O, Nilsen G, Smevik O, Rinck P (1993): Minor structural brain changes in young drug abusers. A magnetic resonance study. Acta Neurol Scand 87: 210-214

Anand BK, Malhotra CL, Singh B, Dua S (1959): Cerebellar projections to limbic system. J Neurophysiol 22:451-458

Berman AF, Berman D, Prescott JW (1978): The effects of cerebellar lesions on emotional behavior in rhesus monkey. In Cooper IS, Riklan M, Snider RS (eds), The Cerebellum, Epilepsy, and Behavior. New York, Plenum Press, pp 277-284

Cohen J (1977): Statistical Power Analysis for the Behavioral Sciences. New York, Academic Press

Courchesne E, Yeung-Courchesne R, Press GA, Hesselink JR, Jernigan TL (1988): Hypoplasia of cerebellar vermal lobules VI and VII in autism. N Engl J Med 318:13491354

Courchesne E, Press GA, Murakami J, Berthoty D, Grafe M, Wiley CA, Hesselink JR (1989): The cerebellum in sagittal plane-anatomic-MR correlation: 1 . the vermis. AJR 153:829-835

Cutting JC (1976): Chronic mania in childhood: case report 
of a possible association with a radiological picture of cerebellar disease. Psychol Med 6:635-642

Donaldson IM, Cuningham J (1983): Persisting neurologic sequelas of lithium carbonate therapy. Arch Neurol 40:747-751

Escalona PR, Early B, McDonald WM, Doraiswamy PM, Shah SA, Husain MM, Boyko OB, Figiel GS, Ellinwood EH, Nemeroff CB, Krishnan KRR (1993): Reduction of cerebellar volume in major depression: A controlled study. Depression 1:156-158

Hamilton NG, Frick RB, Takahashi T, Hopping MW (1983): Psychiatric symptoms and cerebellar pathology. Am J Psychiatry 140:1322-1326

Heath RG, Dempsey CW, Fontana CJ, Myers WA (1978): Cerebellar stimulation: effects on septal region, hippocampus, and amygdala of cats and rats. Biol Psychiatry 13:501-527

Jacobsen LK, Geidd JN, Berquin PC, Krain AL, Hamburger SD, Kumra S, Rapoport JL (1997): Quantitative Morphology of the cerebellum and fourth ventricle in childhood-onset schizophrenia. Am J Psychiatry 154:1663-1669

Joseph AB, Anderson WH, O'Leary DH (1985): Brainstem and vermis atrophy in catatonia. Am J Psychiatry 142:352-354

Jurjus GJ, Weiss KM, Jaskiw GE (1994): Schizophrenia-like psychosis and cerebellar degeneration. Schizophr Res 12:183-184

Kores B, Lader MH (1997): Irreversible lithium neurotoxicity: an overview. Clin Neuropharmacol 20:283-299

Lippmann S, Manshadi M, Baldwin H, Drasin G, Rice J, Alrajeh S (1982): Cerebellar vermis dimensions on computerized tomographic scans of schizophrenic and bipolar patients. Am J Psychiatry 139:667-668

Lucas FR, Goold RG, Gordon-Weeks, Salinas PC (1998): Inhibition of GSK-3 beta leading to the loss of phosphorylated MAP-IB is an early event in axonal remodelling induced by WNT-7a or lithium. J Cell Sci 111:1351-1361

Nasrallah HA, Jacoby CG, McCalley-Whitters M (1981): Cerebellar atrophy in schizophrenia and mania. Lancet 1:1102

Nasrallah HA, McCalley-Whitters M, Jacoby CG (1982): Cortical atrophy in schizophrenia and mania: A comparative CT study. J Clin Psychiatry 43:439-441
Rieder RO, Mann LS, Weinberger DR, vanKammen DP, Post RM (1983): Computed tomographic scans in patients with schizophrenia, schizoaffective, and bipolar affective disorder. Arch Gen Psychiatry 40:735-739

Sax KW, Strakowski SM, Zimmerman ME, DelBello MP, Keck PE Jr, Hawkins JM (1999): Prefrontal cortical volume and attentional performance in bipolar disorder. Am J Psychiatry 156:139-141

Shah SA, Doraiswamy PM, Husain MM, Escalona PR, Na C, Figiel GS, Patterson LJ, Ellinwood EH, McDonald WM, Boyko OB, Nemeroff CB, Krishnan KRR (1992): Posterior fossa abnormalities in major depression: A controlled magnetic resonance imaging study. Acta Psychiatr Scand 85:474-479

Shear PK, Sullivan EV, Lane B, Pfefferbaum A (1996): Mammillary body and cerebellar shrinkage in chronic alcoholics with and without amnesia. Alc Clin Exp Res 20:1489-1495

Soares JC, Mann JJ (1997): The anatomy of mood disordersreview of structural neuroanatomy. Biol Psychiatry 41:86-106

Spitzer RL, Williams JB, Gibbon M, First MG (1990): Structured Clinical Interview for DSM-III-R- Patient edition (SCID-P). New York, Biometrics Research Department, New York State Psychiatric Institute

Strakowski SM, Wilson DR, Tohen M, Woods BT, Douglass AW, Stoll AL (1993): Structural brain abnormalities in first-episode mania. Biol Psychiatry 33:602-609

Strakowski SM, Keck PE, McElroy SL, West SA, Saw KW, Hawkins JM, Kemetz GF, Upadhyaya VH, Tugrul KC, Bourne ML (1998): Twelve-month outcome after a first hospitalization for affective psychosis. Arch Gen Psychiatry 55:49-55

Strakowski SM, DelBello MP, Sax KW, Zimmerman ME, Hawkins JM, Shear PK, Larson ER (1999): Brain magnetic resonance imaging of structural abnormalities in bipolar disorder. Arch Gen Psychiatry 56:254-260

Yadalam KG, Jain AK, Simpson GM (1985): Mania in two sisters with similar cerebellar disturbance. Am J Psychiatry 142:1067-1069

Yates WR, Jacoby CG, Andreasen NC (1987): Cerebellar atrophy in schizophrenia and affective disorder. Am J Psychiatry 144:465-467 